\title{
A simple and soft chemical deaggregation method producing single-digit detonation nanodiamonds
}

Daiki Terada ${ }^{\dagger}$, Frederick T.-K. So ${ }^{\dagger}$, Bodo Hattendorf, Eiji Ōsawa, Masahiro Shirakawa, Ryuji Igarashi* and Takuya F. Segawa*

${ }^{\dagger}$ Equal author contribution

Dr. D. Terada ${ }^{\dagger}$, F. T.-K. So ${ }^{\dagger}$, Prof. M. Shirakawa

Department of Molecular Engineering, Graduate School of Engineering, Kyoto University, Nishikyo-Ku, Kyoto 615-8510, Japan

Dr. B. Hattendorf

Laboratory for Inorganic Chemistry, ETH Zurich, 8093 Zurich, Switzerland

Prof. E. Ōsawa

NanoCarbon Research Institute, AREC, Shinshu University, Ueda, Nagano 386-8567, Japan

Dr. D. Terada ${ }^{\dagger}$, F. T.-K. So ${ }^{\dagger}$, Prof. M. Shirakawa, Dr. R. Igarashi

Institute for Quantum Life Science, National Institutes for Quantum and Radiological Science and Technology, Anagawa 4-9-1, Inage-ku, Chiba 263-8555, Japan

E-mail: igarashi.ryuji@qst.go.jp

Dr. T. F. Segawa

Laboratory for Solid State Physics, ETH Zurich, 8093 Zurich, Switzerland

E-mail: segawat@ethz.ch 
Detonation nanodiamonds (DNDs) are a class of very small and spherical diamond nanocrystals. They are used in polymer reinforcement materials, as drug delivery systems in the field of nanomedicine or as fluorescent biomarkers. Synthesized by detonation, only the final deaggregation step down to the single-digit nanometer size $(<10 \mathrm{~nm})$ unfolds their full potential. All existing deaggregation methods rely on mechanical forces, such as high-power sonication or beads milling. These techniques entail drawbacks such as contamination of the sample and the need for a specialized apparatus. In this paper, we report a purely chemical deaggregation method by simply combining oxidation in air followed by a boiling acid treatment, to produce highly stable single-digit DNDs in a suspension. The resulting DNDs are surface functionalized with carboxyl groups, the final boiling acid treatment removes primary metal contaminants such as magnesium, iron or copper and the nanoparticles remain dispersed over a wide $\mathrm{pH}$ range. Our method can be easily carried out in a standard chemistry laboratory with commonly available laboratory apparatus. This is a key step for many DNDbased applications, ranging from material science to biological or medical applications and opens a way for inexpensive mass production on industrial scale.

Keywords: nanodiamonds, detonation nanodiamonds, deaggregation, surface chemistry 


\section{Introduction}

Detonation nanodiamonds (DNDs) are the smallest nanodiamond crystals, having only a single-digit nanometer diameter $(<10 \mathrm{~nm}$ in size $)$, which can be produced on a large scale via an inexpensive detonation method. ${ }^{[1]}$ In the last two decades, DNDs have received an increasing interest from various fields, such as material or life sciences, due to their extremely small and uniform particle size as well as outstanding physical and chemical properties. As an $s p^{3}$-carbon nanomaterial, DNDs possess remarkable hardness, high refractive index and high thermal conductivity. ${ }^{[2]}$ Their application has been widely expanded towards polymer reinforcement materials, ${ }^{[3]}$ lubricants, ${ }^{[2,4,5]}$ polishing materials, ${ }^{[6]}$ antioxidants ${ }^{[7,8]}$ or sunscreen. ${ }^{[8,9]}$ Among these applications, DNDs as polymer reinforcement materials have been particularly well-investigated. By forming nanocomposites of a polymer matrix and DNDs, nanodiamonds can provide a highly tailorable combination of properties such as superior mechanical, electric, optical, and thermal properties from the diamond structure, combined with rich surface chemistry and high flexibility for the rational design of the DNDmatrix interface. ${ }^{[3]}$ DNDs are also being regarded as promising carriers in drug delivery systems (DDS), as candidates for disease diagnosis and therapy, as well as imaging probes in the biomedical field thanks to their high biocompatibility. ${ }^{[10-13]}$ Due to their large surface-tovolume ratio, DNDs have a high drug loading capacity, where the molecules can be attached onto the nanoparticle surface through covalent conjugation or physical adsorption. DNDs enable prolonged drug retention time by a factor of up to 10 compared to unmodified drugs, resulting in a great enhancement of the chemotherapeutic efficacy. ${ }^{[14]}$ As a result, DNDs have been promising candidates for in vivo applications, applied to a wide range of species for biomedical research, including mice,${ }^{[15]}$ monkeys $^{[16]}$ and humans. ${ }^{[17]}$ In 2017, DNDs were successfully embedded in a thermoplastic biomaterial for the root canal therapy in human, revealing a clinically applicable platform of composite biomaterials in the field of nanomedicine ${ }^{[17]}$ Last but not least, despite their very small size, DNDs can be a crystal host 
for nitrogen-vacancy (NV) centers. ${ }^{[18]} \mathrm{NV}$ centers are a unique color center in diamond with the possibility for optically-detected magnetic resonance (ODMR) for potential applications in bioimaging ${ }^{[19]}$, and can be artificially enriched in DNDs. ${ }^{[20]}$

To utilize the full potential of DNDs, it is extremely important to deaggregate the large DND clusters down to the single-digit size elementary particles and then to stabilize them in a suspension. For example, in filler nanomaterials, the physical properties of the polymer nanocomposite would be drastically enhanced using single-digit nanodiamonds, due to their high surface-to-volume ratio. Single-digit nanoparticles can be useful for targeting and for chemical coupling to biomolecules such as proteins or nucleic acids due to their comparable small sizes. Therefore, producing stable single-digit DNDs in a suspension is highly desirable for practically all DND-based applications.

A tentative explanation for the unusually strong DND aggregation is the highly heterogenous chemical surface containing various functional groups, such as carboxyl, hydroxyl, or lactone groups, which may lead to multiple hydrogen bonds and even covalent bonds between adjacent DND particles. ${ }^{[21]}$ A more homogeneous presence of carboxyl groups on the DND particle surface however would enhance the colloidal stability. Carboxyl groups on the DND surface are formed by oxidation, including air-oxidation ${ }^{[22]}$ and acid treatment. ${ }^{[2]}$ A series of other studies sees aggregation mainly triggered by the presence of ions, especially polyvalent metal ions like copper or iron. ${ }^{[23-25]}$ The experiments strongly supported the model, where individual nanodiamonds are coupled through bridging of metal ions and carboxyl groups on the DND surface. ${ }^{[23-25]}$ Furthermore, a theoretical study highlighted that the DND (100) crystal surfaces have a strongly positive electrostatic potential, while the (111) facets, have often a negative one due to graphitization. ${ }^{[26]}$ In this picture, the electrostatic attraction between DND particles would then lead to strong aggregation. ${ }^{[26]}$ To date, the aggregation mechanisms are still under continuous debate and no consensus is yet reached. 
Interestingly enough, a first deaggregation of DNDs down to a particle size of down to $4.6 \pm$ $0.8 \mathrm{~nm}$ (DLS, containing $99.4 \mathrm{wt} \%$ of the peak $)^{[27]}$ was only achieved back in 2005 by Krüger et al. using stirred-media milling with micron-sized ceramic beads. ${ }^{[28]}$ Subsequent approaches included $\mathrm{ZrO}_{2}$-assisted wet beads milling, ${ }^{[29,30]}$ bead-assisted sonic disintegration (BASD), ${ }^{[30-32]}$ salt- and sugar-assisted ball milling, ${ }^{[33-35]}$ and salt-assisted ultrasonic deaggregation (SAUD) ${ }^{[21]}$ (all summarized in Table 1). Although these techniques are able to break the strongly aggregated DNDs into single-digit $(<10 \mathrm{~nm}$ in size) nanoparticles, they often have critical disadvantages regarding their use in applications, especially in the field of biology. Among all previously mentioned methods, $\mathrm{ZrO}_{2}$-assisted wet milling and BASD are the most common techniques. For BASD, micrometer-sized $\mathrm{ZrO}_{2}$ particles are accelerated with shock waves generated by the ultrasonic tip. The resulting impact and shear forces from the particle collisions breaks the strongly aggregated DNDs and create single-digit DNDs in a range between ca. $\mathrm{pH} \approx 3-6 \cdot{ }^{[31,32]}$ However, the BASD products are accompanied by the difficult-to-remove $\mathrm{ZrO}_{2}$ debris contamination and may lead to formation of $\mathrm{C}-\mathrm{C}$ double bonds and $\mathrm{OH}$ functional groups on the DND surface after prolonged BASD. ${ }^{[32]}$ Although $\mathrm{ZrO}_{2}$ particles debris can be reduced from $17 \mathrm{wt} \%$ to $9.7 \mathrm{wt} \%$ by phosphoric acid treatment and further washing/centrifugation cycles, it is difficult to completely remove the contamination due to its high chemical resistance and similar particle size as the DNDs. ${ }^{[32]}$ To address these drawbacks, salt- and sugar-assisted ball milling ${ }^{[33-35]}$ were developed as alternative techniques utilizing water-soluble crystals such as sodium chloride or sucrose, which can be easily removed from the DND suspension. ${ }^{[21,33]}$ A specialized milling chamber is indispensable for these techniques, which is expensive and needs dedicated expertise to operate it. The single-digit DNDs are only obtained at $\mathrm{pH} \approx 11$ due to the surface profile changes that occurred during the milling process. ${ }^{[33]}$ As an improved method, SAUD was developed in 2016, using only a homogenizer, glass, metal, or plastic containers and crystalline salts such as $\mathrm{NaCl}$ or $\mathrm{KCl}$, enabling a simpler production of single-digit DNDs. ${ }^{[21]}$ 
By combining the benefits of both, BASD and salt- and sugar-assisted ball milling, SAUD simplifies the disintegration process and overcomes the contamination originating from the beads and does not require a specialized milling chamber. SAUD has, in many perspectives, great advantages over other deaggregation techniques. However, some problems are yet to be solved. Primary metal contaminants are left in the suspension, which may hinder various applications based on these dispersed DNDs. Especially, metal ions adsorbed on the nanodiamonds surface have proofed to be a source of cytotoxicity. ${ }^{[36]}$ The experimental setup is greatly simplified, but the need for a high-power sonotrode $(250 \mathrm{~W})$ remains. The strong ultrasound power might damage the sonotrode, which may introduce new contamination into the sample. ${ }^{[32]}$

To summarize, conventional mechanical techniques in fabricating a single-digit DND suspension have the following three issues: (1) contaminations (beads, sodium chloride, metals such as iron, etc.), (2) difficulty in controlling the DND surface profile and (3) need for a dedicated apparatus. In this report, we propose a novel, facile, inexpensive and highly scalable technique to create monodisperse single-digit DND suspension with strongly reduced contamination, using only chemical means: air-oxidation at $425^{\circ} \mathrm{C}$ for 5 hours followed by a boiling acid treatment in $\mathrm{HNO}_{3} / \mathrm{H}_{2} \mathrm{SO}_{4}\left(1: 3 \mathrm{v} / \mathrm{v}\right.$ ratio) at $130{ }^{\circ} \mathrm{C}$ for 3 days (Scheme 1 and Figure 1). As a work up, larger particles are separated by ultracentrifugation. These two-step reaction is inspired by our previous work, ${ }^{[20]}$ where electron irradiation of nanodiamonds followed by the same boiling acid treatment lead to monodisperse single-digit nanodiamonds. Since the electron irradiation had oxidized the nanodiamonds' surface, ${ }^{[20]}$ we have replaced this step with a common oxidation step in air. Our new approach produces not only DNDs functionalized with the bioactive carboxyl groups, guaranteeing a high reactivity. It also successfully removes the initial metal contaminations such as magnesium, iron or copper from the surface of DNDs, generated during the detonation production synthesis. Our technique can 
be easily carried out in any common chemistry or nanotechnology laboratory and significantly lowers difficulty and cost with respect to all previously developed mechanical methods. Last but not least, it is ideally suited for industrial upscaling.

\section{Results and Discussion}

\subsection{Characterization of DNDs: DRIFTS, XPS and LA-ICPMS}

The samples were characterized by diffuse reflectance infrared Fourier transform spectroscopy (DRIFTS) to confirm the organic surface chemical changes during the airoxidation and boiling acid treatment steps (Figure 2(a)). While C-H vibrations (3000-2850 $\left.\mathrm{cm}^{-1}\right)$ disappeared after the air-oxidation, $\mathrm{C}=\mathrm{O}$ and $\mathrm{C}-\mathrm{O}$ vibrations $\left(1805\right.$ and $940-1370 \mathrm{~cm}^{-1}$, respectively) were enhanced, indicating the formation of oxygen-containing derivatives such as carboxylic acids, lactones, anhydrides, hydroxyl groups, cyclic ketones, and saturated structures. ${ }^{[2,37]}$ After the boiling acid treatment, the $\mathrm{C}=\mathrm{O}$ vibration peak was downshifted to $1780 \mathrm{~cm}^{-1}$, which supports the formation of carboxyl groups on the DND surface. ${ }^{[38]}$ By consulting a complementary analytical method, we measured X-Ray photoelectron spectroscopy (XPS) of all nanodiamond samples. In the first reaction step of the airoxidation, the original findings by Osswald et al. ${ }^{[22]}$ were confirmed: An equal $s p^{2}$ vs. $s p^{3}$ carbon content was fully converted into $s p^{3}$ with no $s p^{2}$ carbon left (see Table 2 and Figure $\mathrm{S} 2$ ). In parallel, an increase of $\mathrm{C}=\mathrm{O}, \mathrm{C}-\mathrm{O}, \mathrm{C}-\mathrm{N}$ bonds, graphitic carbon and oxygen was observed. In contrast, the second reaction step of the boiling acid treatment did not merely change the ratios of the different carbon groups. Only a slight increase of the $s p^{3}$ carbon going along with a decrease in the graphitic carbon was confirmed. The final product showed a contamination with sodium, which is introduced by the $\mathrm{NaOH}$ washing step after the boiling acid treatment.

To cover the inorganic part in the chemical analysis, we performed laser ablation inductively coupled plasma mass spectrometry (LA-ICPMS $)^{[39]}$ with a special focus on metal impurities 
in the DND samples. Twenty-four isotopes from different elements were measured and their intensity ratios relative to the carbon-13 signal were determined as a measure for the relative abundance of the respective elements in the material. Due to lack of a suitable calibration standard only the relative abundance of a specific element across the different samples can be concluded from the data. The results are visualized in Figure 4, where the intensity ratios of all isotopes are normalized to one for the starting material "DND" (numerical values can be found in Table S1). After the first reaction step of the air-oxidation, almost all elements were observed at intensity ratios two to three times higher than in the starting material. Rather than a global contamination, this result is more likely explained by a carbon loss during air oxidation. This quantitatively agrees well with the average weight loss during this experimental step. The combustion leads to partial loss of carbon as $\mathrm{CO} / \mathrm{CO}_{2}$ while the metal constituents mostly remain in the solid. Their enrichment relative to the matrix element carbon results in a comparable increase in intensity ratios. Notable exceptions are $\mathrm{V}, \mathrm{Zr}$ and Hf with up to five times increase in intensity ratios, probably by contamination during the combustion process. After boiling the material subsequently in $\mathrm{H}_{2} \mathrm{SO}_{4} / \mathrm{HNO}_{3}$, substantially lower intensity ratios were obtained for most isotopes indicating that abundance of these elements could be successfully reduced in the cleaning step. Namely for the elements $\mathrm{Na}, \mathrm{Al}$, $\mathrm{K}, \mathrm{Ca}, \mathrm{Na}, \mathrm{Cr}, \mathrm{Mn}, \mathrm{Fe}, \mathrm{Co}, \mathrm{Cu}, \mathrm{Zn}$, the average concentration was reduced by more than one order of magnitude. This strongly supports that the second step of the deaggregation is linked to the removal of these metal ions, which hold the individual DND particle together via their chelating surface functional groups. ${ }^{[23-25]}$ The extraction was however not equally efficient and $\mathrm{Si}, \mathrm{Ti}, \mathrm{V}, \mathrm{Sb}$ and $\mathrm{Ba}$ in particular did not show substantial depletion, while $\mathrm{B}$ appeared at even higher levels than after the first reaction step. A similar tendency for the studied elements were previously obtained in a elemental analysis after microwave-assisted purification of detonation nanodiamonds using acid reagents. ${ }^{[40]}$ This appears to result from a combination of low solubility in the acid mix ( $\mathrm{Si}, \mathrm{Sb}, \mathrm{Ba})$ and potentially the entrainment of $\mathrm{B}$ 
2020-11-23

as contaminant of the reagents used. A comparison with the commercial NanoAmando (NanoCarbon Research Institute) detonation nanodiamonds shows, that these BASD (using Zirconia beads) dispersed DNDs have about two orders of magnitude higher $\mathrm{Zr}$ contamination than our dispersed "DND_AirOx_Acid" (see SI Table S1). Earlier studies using inductively coupled plasma-atomic emission spectroscopy (ICP-AES) measured a $\mathrm{Zr}$ contamination of about $3 \mathrm{mg} / \mathrm{g}$ in NanoAmando samples. ${ }^{[25]}$

\subsection{Morphology and Crystal Structure of DNDs: TEM with SAED}

To confirm the diamond crystal structure, transmission electron microscopy (TEM) imaging was conducted on the different DND samples (Figure 3). Figure 3(a) shows large aggregates in "DND" at a low magnification. Figure 3(b) and (c) shows the "DND_AirOx_Acid*" after air-oxidation and boiling acid treatment in a much more uniform and dispersed state. The selected area electron diffraction (SAED) pattern as an inset of Figure 3(b)), with three rings representing (111), (220), and (311) planes of processed DNDs, confirming the DND particles retained their nanodiamond crystal structure. Under high magnification in Figure 3(c), individual nanoparticles in final DNDs with a diameter from 2 to $10 \mathrm{~nm}$ were observed. The results demonstrated that our method does not influence the crystal structure of the DNDs.

\subsection{Colloidal Stability and Redispersibility of DNDs: DLS}

To evaluate the colloidal stability, all samples were investigated by dynamic light scattering (DLS) and zeta potential measurements after dispersion in water. "DND" showed a single peak in DLS at $2.19 \pm 0.15 \mu \mathrm{m}$ and a zeta potential around $0 \mathrm{mV}$ (see Figure 2 (b) and (c)) indicating strongly aggregated DNDs. The suspension of the "DND_AirOx" shows two broad peaks centered at $150 \mathrm{~nm}$ and a broad peak with a maximum around $5 \mu \mathrm{m}$ with a negative zeta potential of $-27 \mathrm{mV}$. The major volume fraction of the "DND_AirOx" was part of a broad $150 \mathrm{~nm}$ DLS peak, suggesting the oxygen species on the DND surface enhanced the 
hydrophilicity and therefore enhanced the dispersibility in water compared to DND. The simultaneous decrease of the zeta potential supported the presence of oxygen-containing functional groups, which led to an increase in hydrophilicity and hence particle size reduction. After the boiling acid step, the sample reached a monodisperse state with a hydrodynamic size of $3.57 \pm 0.04 \mathrm{~nm}$ in DLS with a polydispersity index (PDI) of 0.33 . The further reduction of the zeta potential down to $-40 \mathrm{mV}$ is attributed to the increased number of carboxyl groups. Keeping the results from LA-ICPMS measurements in mind, we believe that the strong reduction of the contamination from many metal elements is at least as important as the organic chemical change on the nanodiamond surface. The results demonstrated that these two chemical steps are needed to make single-digit nanodiamonds with high yield. The boiling acid treatment alone does not disperse the starting material "DND" (see Figure S1). According to these observations, we strongly believe the stable single-digit state of the sample is contributed by the removal of metal impurities and the presence/formation of the carboxyl groups on the surface of DNDs. The highly negative zeta potential is linked to be the presence of carboxyl groups because the $\mathrm{COO}^{-}$groups are formed in the water at $\mathrm{pH}=5-12 .{ }^{[41]}$

While all measurements discussed above were performed at $\mathrm{pH}=8.75$, we further studied the dependence of the colloidal stability as a function of $\mathrm{pH}$ (Figure 5(a)). Aggregation behavior on varying $\mathrm{pH}$ reflects the DND surface profile with functional groups on complex surfaces exhibiting a range of $\mathrm{pKa}$ values. The single-digit window of the nanoparticle size was $5 \leqq$ $\mathrm{pH}<11$ with zeta potentials of around $-30 \mathrm{mV}$ (Figure 5(a)). Strong aggregation was only observed below $\mathrm{pH}$, which is caused by a loss of surface charge due to protonation of the carboxylate and lactone groups in particular. As a result, the negatively-charged DNDs showed good colloidal stability over a wide range of $\mathrm{pH}$ values, covering both, alkaline and acidic conditions, in comparison to BASD DNDs (positively-charged DNDs) as reported before. ${ }^{[42]}$ Moreover, negatively-charged DNDs were shown to have a stronger aggregation resistance at higher ionic strength in comparison to positively-charged DNDs. ${ }^{[42]}$ 
Finally, we observed that the freeze-dried air-oxidized DNDs after boiling acid were redispersed in water down to a single-digit size of $3.51 \pm 0.11 \mathrm{~nm}$ with a PDI of 0.312 (Figure 5(b)). This is a remarkable difference to freeze-dried BASD DNDs, which cannot be redispersed in the same way and do show aggregation with particle sizes $1.75 \pm 0.21 \mu \mathrm{m}$ (Figure 5(b)).

\section{Conclusion}

We report a facile, inexpensive, and highly scalable chemical approach for the production of monodisperse, single-digit DNDs with reduced metal contamination levels. Our method, oxidation in air followed by a boiling acid $\left(\mathrm{HNO}_{3} / \mathrm{H}_{2} \mathrm{SO}_{4}, 1: 3 \mathrm{v} / \mathrm{v}\right.$ ratio) acid treatment is "purely chemical": no high-power sonication or bead milling technique is used for the deaggregation. The recipe could serve both, industrial and research purposes, providing $s p^{3}$ carbonmaterials, which could improve the excellent physical/chemical properties in nanocomposites and open up new opportunities in the area of biomedical research as DNDs in DDS, diagnosis and therapy.

The chemical treatments introduce carboxyl groups to the DNDs' surface, which help stabilizing the single-digit nanodiamonds in a suspension by maintaining a high negative surface charge. Using the platform of carboxyl enriched nanodiamond surface, the DND could be widely functionalized with readily available chemical nanodiamond modification techniques, ${ }^{[3]}$ leading to the control of the dispersibility in various solvents and the formation of covalent bonds to polymers of choice. ${ }^{[43]}$ The produced DNDs are likely to solve two major issues in polymer nanocomposite: a) the poor dispersibility (aggregated particles create defects in polymer nanocomposite) and b) the weak interface due to the difficulty in making covalent bonds between nanodiamonds and the matrix. Thereby superior composites with very high mechanical, thermal, and other properties can be realized. ${ }^{[3]}$ Also, carboxyl group enriched DNDs can be immediately conjugated with drugs and biomolecules for in-cell 
targeting through carbodiimide crosslinking reactions, which would bring a great advantage for using them in bio-applications. ${ }^{[44]}$ In addition, the single-digit nanodiamonds show excellent colloidal stability over a wide $\mathrm{pH}$ range and large ionic strengths. This gives more freedom for the choice of chemical reactions by protecting the nanodiamonds from aggregation.

LA-ICPMS measurements showed that the boiling acid treatment strongly reduces various metal contaminations generated in the detonation synthesis process (see Figure 3). We believe that this "inorganic clean-up" is the key step to reach the single-digit DND core size by breaking up the inter-particle linkers consisting of metal chelates. Unlike for other mechanical methods, DNDs dispersed by our purely chemical method, can be simply redispersed in water even after freeze-drying.

It is striking that the DLS size distributions of our "DND", "DND_AirOx" and "DND_AirOx_Acid", correspond remarkably well with the "Secondary aggregates", "Core agglutinates/Primary aggregates" and "Primary particles" as described earlier by Ōsawa, when using his bead-milling strategy. ${ }^{[27]}$ Continuing the analogy, our "oxidation in air" would take the place of "intense sonication" (deaggregation down to 100-200 nm), while our "boiling acid treatment" would play the role of the "beads milling" (down to $4.6 \pm 0.8 \mathrm{~nm}$ by DLS, containing $99.4 \mathrm{wt} \%$ of this peak). ${ }^{[27]}$ Such a comparison of the deaggregation method could further help to understand the mechanistic details of the different approaches. In summary, the purely chemical technique to successfully produce single-digit nanodiamonds presents solutions for three central problems in the field: (1) strong reduction of metal contamination originating from the detonation synthesis (while avoiding new contaminations through beads milling etc.); (2) creation of a highly negatively-charged DND surface with enhanced carboxylic acid groups; (3) no need for specialized setups such as bead mill or high-power homogenizer. We strongly believe that the use of our single-digit DNDs 
should provide a standard method for academia and industry, not only for nanocomposite materials, or bioapplications, but for a variety of applications, that are still to be discovered.

\section{Experimental Section}

Materials: $170 \mathrm{~nm}$ DND +15 mV Positive Zeta $1.7 \%$ ash (NDStandard100g) was purchased from Adamas Nanotechnologies, United States. NanoAmando Aqueous Colloid Solution (NanoCarbon Research Institute, Ltd., Ueda, Japan) with particle size $3.2 \pm 0.6 \mathrm{~nm}$ was used as BASD treated control sample. Nitric acid (60\%) and sulphuric acid (95.0+\%) were purchased from Fujifilm Wako Pure Chemical Corporation, Japan.

Characterization: DRIFT spectra were acquired by using FT/IR-6600 (JASCO) instrument equipped with a diffuse reflectance accessory (DR PRO410-M) with a resolution of $4 \mathrm{~cm}^{-1}$. The DND samples and $\operatorname{KBr}(9: 1, w / w)$ were ground using a mortar and pestle, and then heated at $150^{\circ} \mathrm{C}$ under vacuum for $5 \mathrm{~h}$ to remove the adsorbed water. Measurements and the DRIFT spectra processing were conducted, as previously reported.$^{[20]}$ XPS was measured on a KRATOS ULTRA2 (Shimadzu Corp., Japan) with a Pass Energy of $20 \mathrm{eV}$ and a sample area of $300 \times 700 \mu \mathrm{m}$, while the charge neutralizer was on. The binding energy was calibrated to $\mathrm{N}$ $1 \mathrm{~s}(\mathrm{C}-\mathrm{N})=399.5 \mathrm{eV}$. For the spectral decomposition of the $\mathrm{C} 1 \mathrm{~s}$ signal, an energy difference between $\mathrm{sp}^{2}$ and $\mathrm{sp}^{3}$ of $1.1 \mathrm{eV}$ was assumed. The $\mathrm{C}-\mathrm{O}, \mathrm{C}-\mathrm{N}$ and the $\mathrm{C}=\mathrm{O}$ signals had an energy of 1.5 and $2.6 \mathrm{eV}$ higher than the $\mathrm{sp}^{3}$ signal. LA-ICPMS ${ }^{[39]}$ was performed at the Laboratory of Inorganic Chemistry at ETH Zurich. The samples were dried for $24 \mathrm{~h}$ at 100 mbar and $105^{\circ} \mathrm{C}$, homogenized and finally pressed to pellets $(13 \mathrm{~mm}$ diameter, $4-6 \mathrm{~mm}$ height) under a load of 10 tons for 10 minutes. The pressed pellets were mounted on a microscope slide using double-faced adhesive tape. The mounted samples were place in the ablation cell of a laser ablation system (GeoLas Q, Coherent, Göttingen ${ }^{[45]}$ ) connected to a sector field ICPMS (Element 2, Thermo Scientific, Bremen). The samples were ablated using a fluence of $8.5 \mathrm{~J} / \mathrm{cm}^{2}$, a pulse repetition rate of $5 \mathrm{~Hz}$ and using a crater diameter of $120 \mu \mathrm{m}$. 
All samples were analyzed in time resolved mode using $10 \mathrm{~ms}$ integration time per isotope per MS scan. Analyses comprised acquisition of the instrumental background signals for 30 seconds, after which the laser was started and the ablated material analyzed for $60-90$ seconds. The ablation was carried out as single-spot ablation whereby the material is being removed from successively deeper regions below the surface of the pressed pellets. Transient signals thus resemble a depth profile, covering approximately $20 \mu \mathrm{m}$ in depth. Most isotopes' ion signals were recorded using the most sensitive mode of the ICPMS using a mass resolving power (MRP, $\mathrm{m} / \Delta \mathrm{m})$ of 300 . In order to minimize artifacts from spectral interferences however, several isotopes elements were recorded in a second sequence at a mass resolving power of 4000 (see Table S1). Data evaluation followed the protocol described by Longerich et al. ${ }^{[46]}$ with the exception that only qualitative data were collected because of the lack of a suitable calibration standard. For LA-ICPMS, the DND sample after boiling acid, but before $\mathrm{NaOH}$ washing ("DND_AirOx_Acid") and centrifugation step was taken. This explains the discrepancy between the Na content obtained from XPS and LA-ICPMS. Hydrodynamic size distributions and zeta potentials of the samples were performed with a Malvern Zetasizer Nano instrument (Malvern Panalytical Ltd.), "DND”, "DND_AirOx”, "DND_AirOx_Acid*” were measured at $0.9,0.85$ and $17 \mathrm{mg} / \mathrm{mL}$ respectively. TEM images were taken by a JEM2200FS+ CEOS CETCOR (JEOL) instrument at $200 \mathrm{kV}$ acceleration voltage and $0.1 \mathrm{~nm}$ spatial resolution. Samples dispersed in Milli-Q water of $0.5 \mathrm{mg} / \mathrm{mL}$ were dried on a germanium $(\mathrm{Ge})$ film with a thickness of $10 \mathrm{~nm}$, subsequently cleaned with a JIC-410 Ion Cleaner (JEOL) at $300 \mathrm{~V}$ setting. Air-Oxidation: Raw detonation nanodiamond powder (DND) was first ground using mortar and pestle, then transferred into a ceramic crucible for surface oxidation in air at $425{ }^{\circ} \mathrm{C}$ for 5 hours (AMI-2 Oven, NITTO KAGAKU CO., LTD). The oven temperature was confirmed using an infrared thermometer (AD-5616, A\&D Company, Limited). 
Boiling Acid Treatment: $300 \mathrm{mg}$ of air-oxidized raw nanodiamond powder (DND_AirOx) was mixed with $50 \mathrm{~mL}$ of nitric acid and sulphuric acid mixture (1:3 v/v ratio) in an ice-bath sonicator (Cosmo Bio, Bioruptor UCS-200TM, on/off = 30s/30s) for 10 minutes. Next, the mixture was magnetically stirred and heated at $130{ }^{\circ} \mathrm{C}$ for 3 days under reflux inside the fume hood. The reaction mixture was then diluted and cleaned with Milli-Q water 3 times by centrifugation at 150,000 RCF for 45 minutes (Beckman Optima Ultra-centrifugation, TLA110 rotor, and TOMY Digital Biology UR-21P handy sonicator) to remove the remaining acid in the solution. The sample was treated with $1 \mathrm{M} \mathrm{NaOH}$ solution at $90^{\circ} \mathrm{C}$ for 2 hours, subsequently cleaned with Milli-Q water at the above-mentioned centrifugation parameters until no further de-aggregation was observed (in case of $\mathrm{NaOH}$ treatment, the solution was immediately redispersed into $1 \mathrm{M} \mathrm{NaOH}$ after 1 ultra-centrifugation). The black dispersed DND pellet was gently rinsed, collected, concentrated along the washes with the same condition. White tiny non-dispersed pellet was observed in the pellet. Therefore, in order to remove aggregated particles, the solution was ultra-centrifuged at 10,000 RCF for 1 hour and only the supernatant was collected.

Colloidal Stability and Ionic Strength Assessment: To exanimate the colloidal stability under different $\mathrm{pH}$, the final raw DNDs product (DND_AirOx_Acid*) $(\mathrm{pH}=8.75)$ was transferred into two small glass vials separately. $0.5 \mathrm{M} \mathrm{HCl}$ solution and $0.5 \mathrm{M} \mathrm{NaOH}$ solution were added to the two vials individually using an autopipette, while magnetically stirred and monitored by pH meter (HORIBA, laqua 9618s) simultaneously. Once the suspension reached the desirable $\mathrm{pH}$, the hydrodynamic size distribution and zeta potential of the solution were measured using a Malvern Zetasizer Nano instrument (Malvern Panalytical Ltd.). To assess the ionic strength, the $\mathrm{KCl}$ solution was introduced to the final raw DNDs solution, with increasing $\mathrm{KCl}$ concentration $\left(0 \mathrm{M}, 10^{-3} \mathrm{M}, 10^{-2} \mathrm{M}, 10^{-1} \mathrm{M}\right)$, and the corresponding hydrodynamic size distribution was measured using the aforementioned apparatus. 
Acknowledgements

D.T. and F.T.-K.S. contributed equally to this work. T.F.S. acknowledges The Branco Weiss Fellowship - Society in Science, administered by the ETH Zurich. The Japan Science and Technology Agency under Precursory Research for Embryonic Science and Technology ( PRESTO, grant numbers JPMJPR18G1) and MEXT Quantum Leap Flagship Program (MEXT Q-LEAP, grant number JPMXS0120330644) are acknowledged for funding. TEM images were acquired at the Kyoto University Nanotechnology Platform by the expertise of Prof. Kurota and Dr. Kiyomura. XPS data was acquired at Shimadzu Corp. by Mr. Watanabe. Profs. Akiyoshi/Mukai, Kyoto University, are kindly acknowledged for the access to their DLS setup. Parts of the TOC figure have been created with Chemix (https://chemix.org).

\section{References}

[1] V. V. Danilenko, Phys. Solid State 2004, 46, 595.

[2] V. N. Mochalin, O. Shenderova, D. Ho, Y. Gogotsi, Nat. Nanotechnol. 2012, 7, 11.

[3] V. N. Mochalin, Y. Gogotsi, Diam. Relat. Mater. 2015, 58, 161.

[4] V. Y. Dolmatov, J. Superhard Mater. 2010, 32, 14.

[5] O. Shenderova, A. Vargas, S. Turner, D. M. Ivanov, M. G. Ivanov, Tribol. Trans. 2014, 57, 1051 .

[6] A. S. Artemov, Phys. Solid State 2004, 46, 687.

[7] T. M. Chen, X. M. Tian, L. Huang, J. Xiao, G. W. Yang, Nanoscale 2017, 9, 15673.

[8] Q. Lin, R. H. J. Xu, N. Yang, A. Karim, X. J. Loh, K. Zhang, ACS Appl. Nano Mater. 2019, 2, 7604 .

[9] O. Shenderova, V. Grichko, S. Hens, J. Walch, Diam. Relat. Mater. 2007, 16, 2003.

[10] H. Huang, E. Pierstorff, E. Osawa, D. Ho, Nano Lett. 2007, 7, 3305. 
[11] A. M. Schrand, H. Huang, C. Carlson, J. Schlager, S. M. Hussain, L. Dai, J. Phys. Chem. B 2007, 111, 1 .

[12] V. Vaijayanthimala, D. K. Lee, S. V. Kim, A. Yen, N. Tsai, D. Ho, H.-C. Chang, O. Shenderova, Expert Opin. Drug Deliv. 2015, 12, 735.

[13] K. van der Laan, M. Hasani, T. Zheng, R. Schirhagl, Small 2018, 14, 1.

[14] E. K. Chow, X.-Q. Zhang, M. Chen, R. Lam, E. Robinson, H. Huang, D. Schaffer, E. Osawa, A. Goga, D. Ho, Sci. Transl. Med. 2011, 3, 73ra21.

[15] Y. Yuan, X. Wang, G. Jia, J. H. Liu, T. Wang, Y. Gu, S. T. Yang, S. Zhen, H. Wang, Y. Liu, Diam. Relat. Mater. 2010, 19, 291.

[16] L. Moore, J. Yang, T. T. H. Lan, E. Osawa, D. K. Lee, W. D. Johnson, J. Xi, E. K. H. Chow, D. Ho, ACS Nano 2016, 10, 7385.

[17] D.-K. Lee, T. Kee, Z. Liang, D. Hsiou, D. Miya, B. Wu, E. Osawa, E. K.-H. Chow, E. C. Sung, M. K. Kang, D. Ho, Proc. Natl. Acad. Sci. 2017, 114, E9445.

[18] B. R. Smith, D. W. Inglis, B. Sandnes, J. R. Rabeau, A. V. Zvyagin, D. Gruber, C. J. Noble, R. Vogel, E. Ōsawa, T. Plakhotnik, Small 2009, 5, 1649.

[19] S. Sotoma, D. Terada, T. F. Segawa, R. Igarashi, Y. Harada, M. Shirakawa, Sci. Rep. 2018, 8,1 .

[20] D. Terada, T. F. Segawa, A. I. Shames, S. Onoda, T. Ohshima, E. Ōsawa, R. Igarashi, M. Shirakawa, ACS Nano 2019, 13, 6461.

[21] K. Turcheniuk, C. Trecazzi, C. Deeleepojananan, V. N. Mochalin, ACS Appl. Mater. Interfaces 2016, 8, 25461.

[22] S. Osswald, G. Yushin, V. Mochalin, S. O. Kucheyev, Y. Gogotsi, J. Am. Chem. Soc. 2006, 128,11635 .

[23] A. I. Shames, A. M. Panich, V. Y. Osipov, A. E. Aleksenskiy, A. Y. Vul', T. Enoki, K. Takai, J. Appl. Phys. 2010, 107, 014318.

[24] A. E. Aleksenskiy, E. D. Eydelman, A. Y. Vul, Nanosci. Nanotechnol. Lett. 2011, 3, 
68.

[25] P. N. Nesterenko, D. Mitev, B. Paull, in Nanodiamonds Adv. Mater. Anal. Prop. Appl. (Ed: J.C. Arnault), Elsevier, 2018, pp. 109-130.

[26] A. S. Barnard, M. Sternberg, J. Mater. Chem. 2007, 17, 4811.

[27] E. Ōsawa, Pure Appl. Chem. 2008, 80, 1365.

[28] A. Krüger, F. Kataoka, M. Ozawa, T. Fujino, Y. Suzuki, A. E. Aleksenskii, A. Y. Vul', E. Ōsawa, Carbon 2005, 43, 1722.

[29] E. D. Eidelman, V. I. Siklitsky, L. V. Sharonova, M. A. Yagovkina, A. Y. Vul', M. Takahashi, M. Inakuma, M. Ozawa, E. Ōsawa, Diam. Relat. Mater. 2005, 14, 1765.

[30] A. Krueger, M. Ozawa, G. Jarre, Y. Liang, J. Stegk, L. Lu, Phys. status solidi 2007, 204, 2881.

[31] M. Ozawa, M. Inaguma, M. Takahashi, F. Kataoka, A. Krüger, E. Ōsawa, Adv. Mater. 2007, 19, 1201.

[32] Y. Liang, M. Ozawa, A. Krueger, ACS Nano 2009, 3, 2288.

[33] A. Pentecost, S. Gour, V. Mochalin, I. Knoke, Y. Gogotsi, ACS Appl. Mater. Interfaces 2010, 2, 3289.

[34] W. Peng, R. Mahfouz, J. Pan, Y. Hou, P. M. Beaujuge, O. M. Bakr, Nanoscale 2013, 5, 5017.

[35] M. Khan, N. Shahzad, C. Xiong, T. K. Zhao, T. Li, F. Siddique, N. Ali, M. Shahzad, H. Ullah, S. A. Rakha, Diam. Relat. Mater. 2016, 61, 32.

[36] Y. Zhu, Y. Zhang, G. Shi, J. Yang, J. Zhang, W. Li, A. Li, R. Tai, H. Fang, C. Fan, Q. Huang, Part. Fibre Toxicol. 2015, 12, 1.

[37] T. Petit, L. Puskar, Diam. Relat. Mater. 2018, 89, 52.

[38] T. Ando, K. Yamamoto, M. Ishii, M. Kamo, Y. Sato, J. Chem. Soc. Faraday Trans. 1993, 89, 3635.

[39] D. Günther, B. Hattendorf, TrAC - Trends Anal. Chem. 2005, 24, 255. 
2020-11-23

[40] D. P. Mitev, A. T. Townsend, B. Paull, P. N. Nesterenko, Diam. Relat. Mater. 2014, $48,37$.

[41] P. Reineck, D. W. M. Lau, E. R. Wilson, N. Nunn, O. A. Shenderova, B. C. Gibson, Sci. Rep. 2018, $8,1$.

[42] C. Bradac, I. Das Rastogi, N. M. Cordina, A. Garcia-Bennett, L. J. Brown, Diam. Relat. Mater. 2018, 83, 38.

[43] J. Neburkova, J. Vavra, P. Cigler, Curr. Opin. Solid State Mater. Sci. 2017, 21, 43.

[44] D. Terada, T. Genjo, T. F. Segawa, R. Igarashi, M. Shirakawa, Biochim. Biophys. Acta - Gen. Subj. 2020, 1864, 129354.

[45] D. Günther, R. Frischknecht, C. A. Heinrich, H.-J. Kahlert, J. Anal. At. Spectrom. 1997, 12, 939 .

[46] H. P. Longerich, S. E. Jackson, D. Günther, J. Anal. At. Spectrom. 1996, 11, 899. 

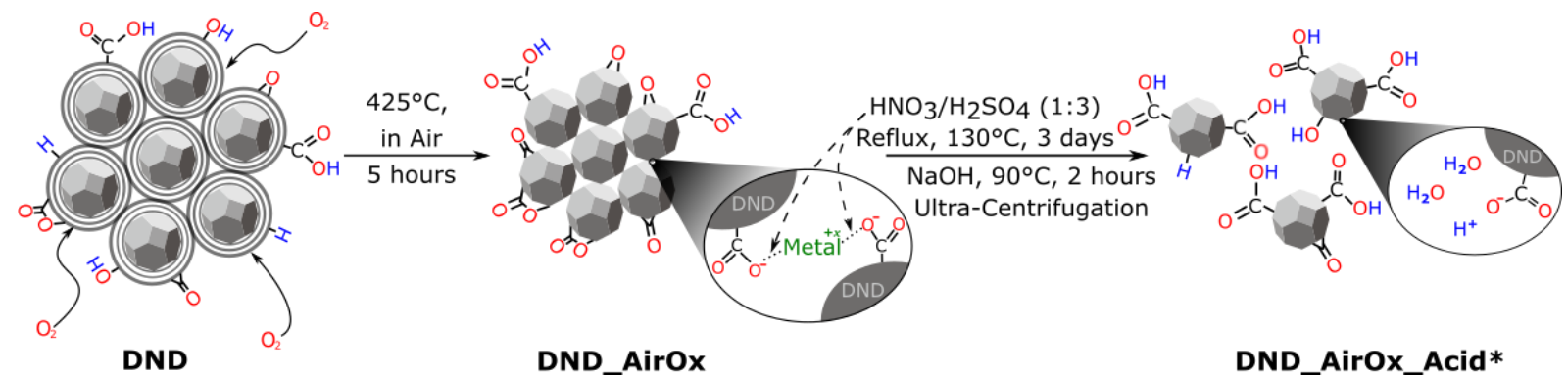

Scheme 1. Reaction scheme for the purely chemical deaggregation of detonation nanodiamonds and their abbreviations used throughout the text (for more details, see Scheme S1).

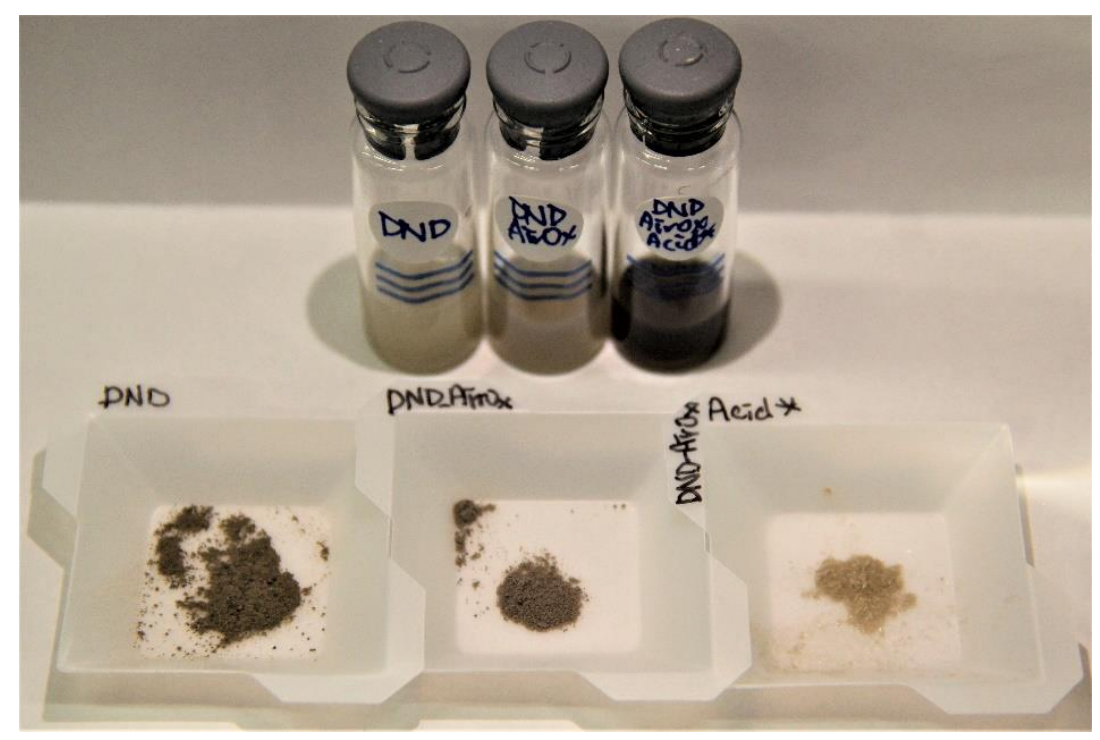

Figure 1. Photograph of suspensions and dried powders of "DND", "DND_AirOx" and "DND_AirOx_Acid*". 


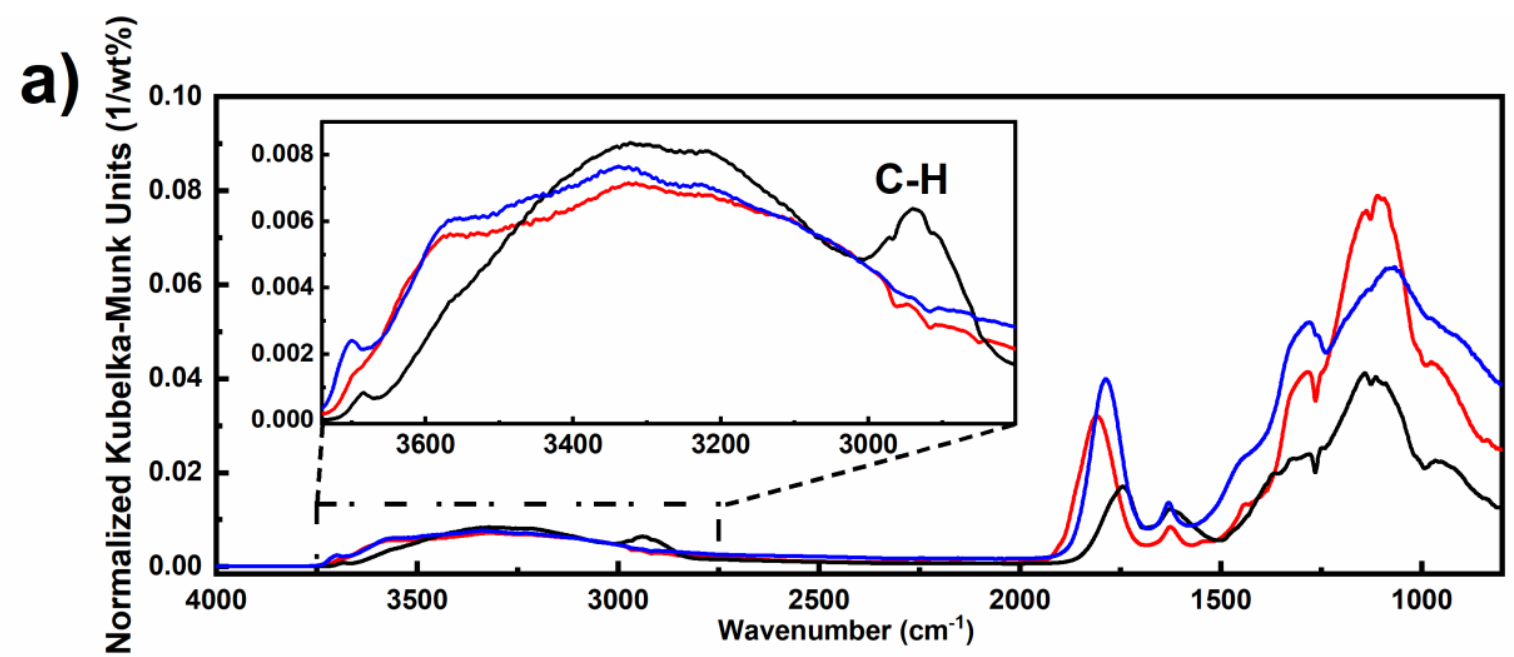

b)

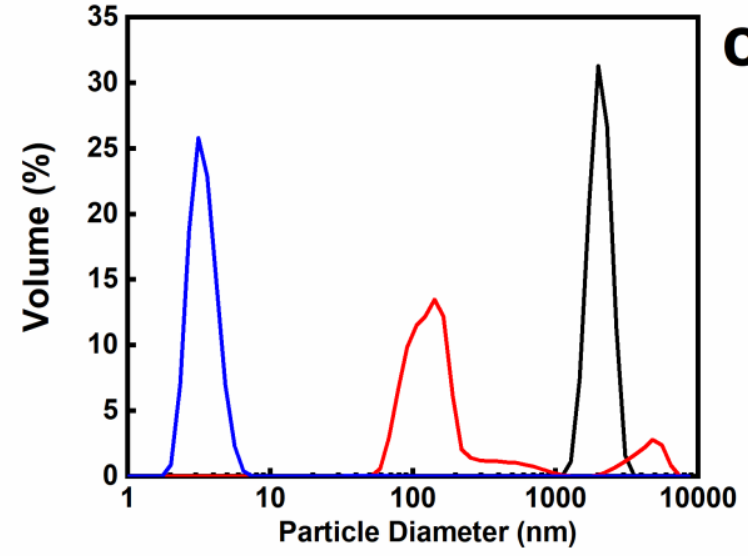

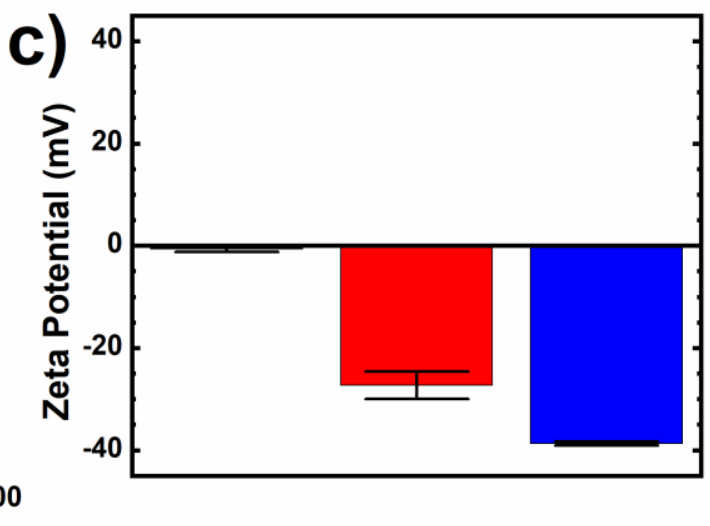

Figure 2. Characterization of "DND" (black), "DND_AirOx" (red) and

"DND_AirOx_Acid*” (blue). (a) DRIFT spectra, (b) DLS size distribution by volume and (c) zeta potentials. 

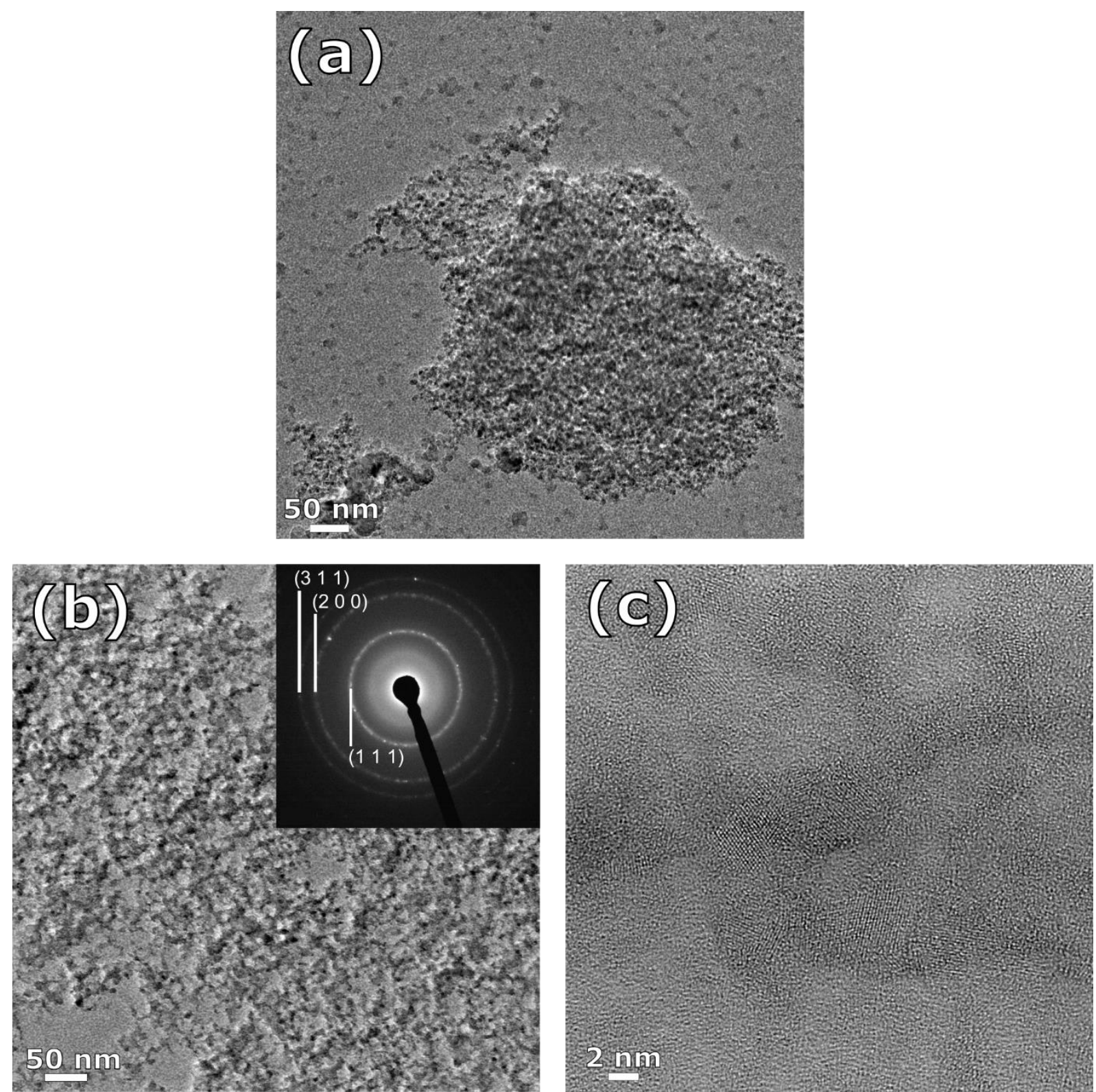

Figure 3. (a) Representative TEM image of the starting material "DND" and (b) the final

"DND_AirOx_Acid*" with the diamond crystal structure confirmed by selected-area electron diffraction (SAED) (inset). (c) Same sample as (b) in a higher magnification. 


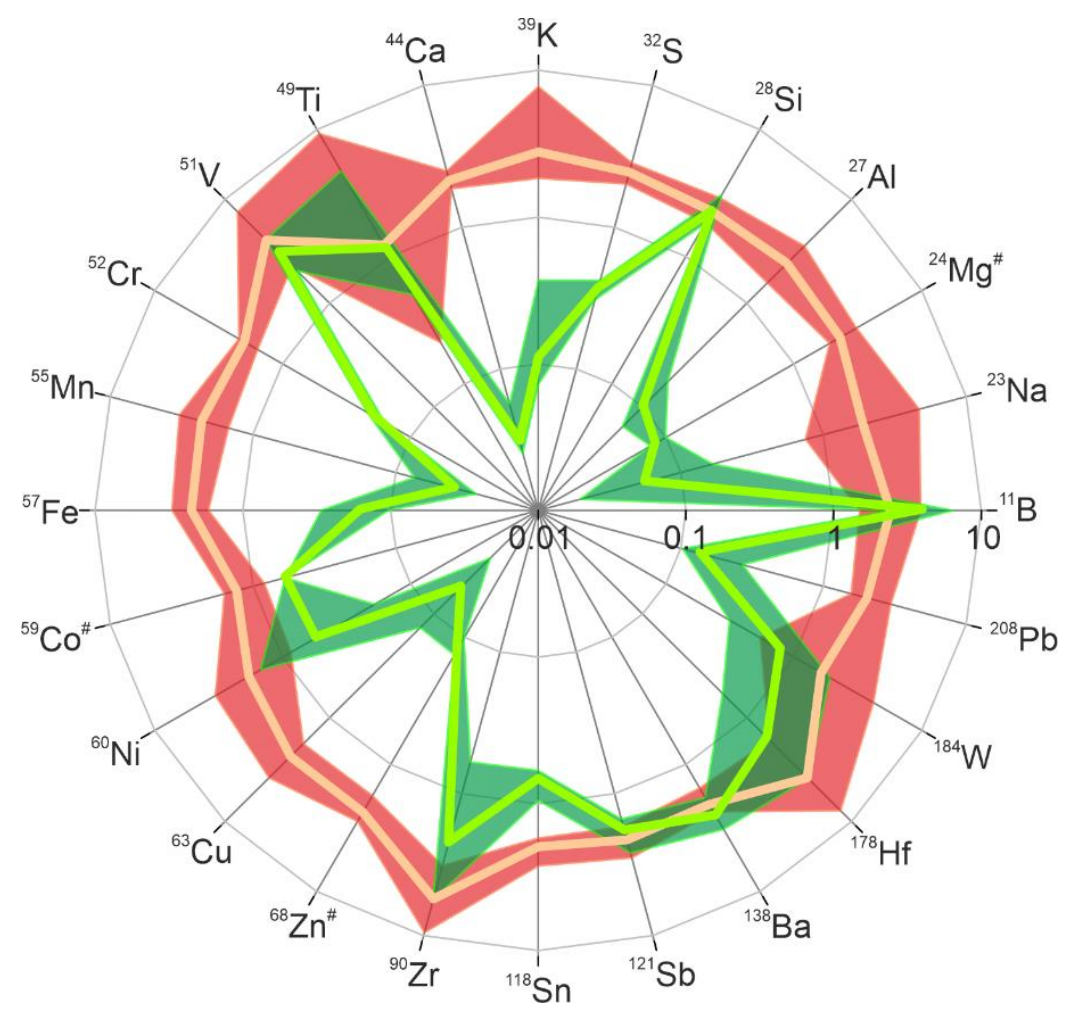

Figure 4. Radar plots, indicating measured intensity ratios for the "DND_AirOx" (in red) and "DND_AirOx_Acid" (in green) with respect to those for the starting material "DND". The orange and green lines show the mean values from five repeat analyses of each sample. The thicker red and green areas, indicate the ranges of the intensity ratios across the five repeats. Values greater 1 indicate a relative increase in the metal/carbon ratio relative to the starting material. Note that the relative intensity ratios are plotted on a log-scale. The ${ }^{59} \mathrm{Co}$ concentration was below the limit of detection (LOD) in sample "DND" and “DND_AirOx_Acid" and ${ }^{24} \mathrm{Mg}$ and ${ }^{68} \mathrm{Zn}$ contents were below LOD in the “DND_AirOx_Acid" sample. In these cases (highlighted with \#), the ratios were calculated using the respective LOD value. 

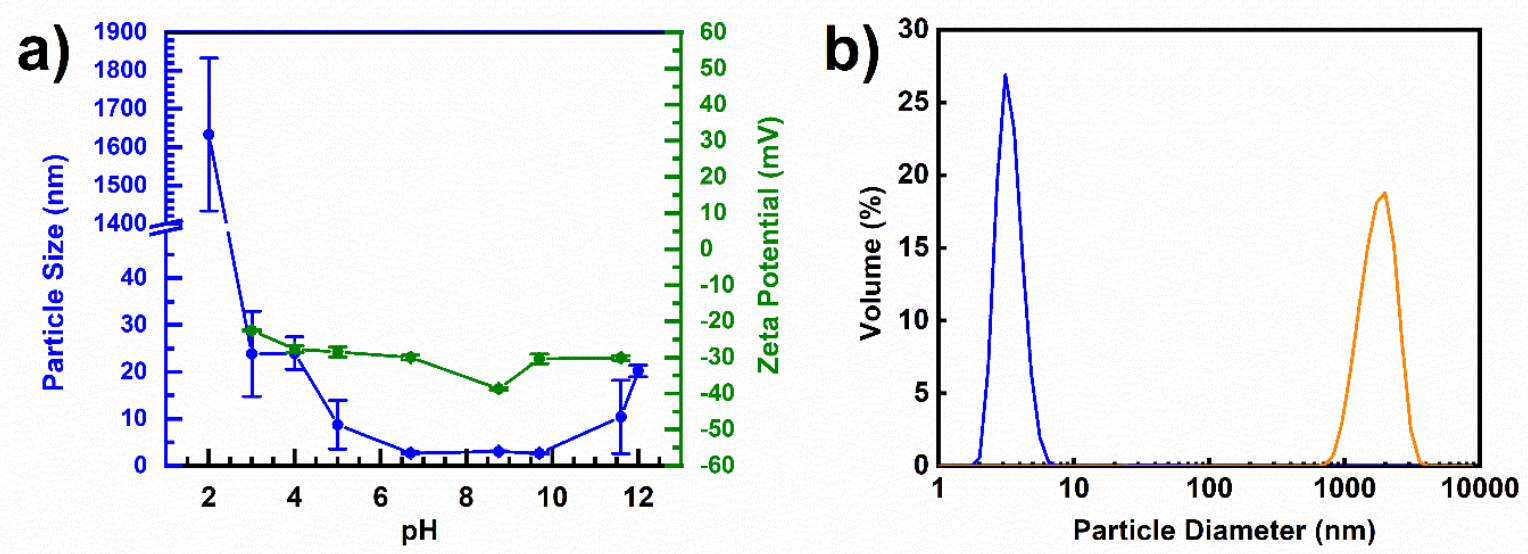

Figure 5. (a) Particle sizes (blue, left axis) and zeta potentials (green, right axis) of "DND_AirOx_Acid*" at various $\mathrm{pH}$ values. The points at $\mathrm{pH}=8.75$ correspond to the data in Figure 2(b) and (c). (b) Representative DLS size distribution of redispersed detonation nanodiamonds in water after freeze-drying: "DND_AirOx_Acid*” (blue) and BASDdispersed detonation nanodiamonds NanoAmando (orange). 


\begin{tabular}{|c|c|c|c|c|c|c|c|c|}
\hline Techniques & $\begin{array}{l}\text { Nature of } \\
\text { the method }\end{array}$ & $\begin{array}{l}\text { Experimental } \\
\text { setup }\end{array}$ & Additive & Process & $\begin{array}{c}\text { Particle } \\
\text { Diameter } \\
(\mathrm{nm})\end{array}$ & $\begin{array}{c}\text { Redispersibility, } \\
\text { particle diameter } \\
\text { (nm) }\end{array}$ & Remarks & References \\
\hline $\begin{array}{l}\text { Bead-assisted } \\
\text { ball milling }\end{array}$ & Mechanical & $\begin{array}{l}\text { dedicated } \\
\text { milling } \\
\text { chamber }\end{array}$ & $\begin{array}{l}\mathrm{SiO}_{2}, \mathrm{ZrO}_{2} \\
\text { microbeads }\end{array}$ & $\begin{array}{l}\text { strong base or } \\
\text { acid treatment } \\
\text { to dissolute } \\
\mathrm{ZrO} 2 \text { debris }\end{array}$ & $\begin{array}{c}4.6 \pm 0.8 \\
{[27]}\end{array}$ & 2300 (after drying) & $\begin{array}{l}\text { Difficult-to- } \\
\text { remove } \\
\text { microbeads } \\
\text { contamination }\end{array}$ & {$[27-30]$} \\
\hline $\begin{array}{l}\text { Bead-assisted } \\
\text { sonic } \\
\text { disintegration } \\
\text { (BASD) }\end{array}$ & Mechanical & $\begin{array}{l}\text { high power } \\
\text { homogenizer } \\
(400-450 \mathrm{~W})\end{array}$ & $\begin{array}{c}\mathrm{ZrO}_{2} \\
\text { microbeads }\end{array}$ & $\begin{array}{l}\text { strong base or } \\
\text { acid treatment } \\
\text { to dissolute } \\
\text { ZrO2 debris }\end{array}$ & $\begin{array}{l}4.8 \text { (for } \\
\text { arylated } \\
\text { DND) }\end{array}$ & $\begin{array}{l}1000-3000 \text { (after } \\
\text { freeze-drying) } \\
2300 \text { (after drying) }\end{array}$ & $\begin{array}{l}\text { Difficult-to- } \\
\text { remove } \\
\text { microbeads } \\
\text { contamination }\end{array}$ & {$[31,32]$} \\
\hline $\begin{array}{l}\text { Salt-assisted } \\
\text { attrition } \\
\text { milling }\end{array}$ & Mechanical & $\begin{array}{l}\text { dedicated } \\
\text { milling } \\
\text { chamber }\end{array}$ & $\begin{array}{c}\mathrm{NaCl} \\
\text { crystals }\end{array}$ & $\begin{array}{l}\text { Acid treatment } \\
\text { to remove iron } \\
\text { and other } \\
\text { metals, and pH } \\
\text { adjustment to } \\
11\end{array}$ & $<10$ & 16-18 (after drying) & $\begin{array}{c}\text { Iron } \\
\text { contamination } \\
\text { comes from } \\
\text { steel balls and } \\
\text { parts of } \\
\text { the mill }\end{array}$ & [33] \\
\hline $\begin{array}{l}\text { Salt-assisted } \\
\text { ultrasound } \\
\text { deaggregation } \\
\text { (SAUD) }\end{array}$ & Mechanical & $\begin{array}{l}\text { high power } \\
\text { homogenizer } \\
(150 \mathrm{~W})\end{array}$ & $\begin{array}{c}\mathrm{NaCl} \\
\text { crystals }\end{array}$ & $\begin{array}{l}\text { Washing/ } \\
\text { Centrifugation }\end{array}$ & $5-10$ & 10-20 (after drying) & $\begin{array}{c}\mathrm{NaCl} \\
\text { contamination }\end{array}$ & [21] \\
\hline $\begin{array}{c}\text { Purely } \\
\text { chemical } \\
\text { treatment }\end{array}$ & chemical & $\begin{array}{l}\text { common } \\
\text { chemistry } \\
\text { instruments } \\
\text { (flask, etc.) }\end{array}$ & & $\begin{array}{l}\text { Washing/ } \\
\text { Centrifugation }\end{array}$ & 3-5 (DLS) & $\begin{array}{c}\text { 3-8 (after freeze- } \\
\text { drying) }\end{array}$ & Low Impurities & This work \\
\hline
\end{tabular}

Table 1. Comparison of existing deaggregation techniques (modified from Ref. ${ }^{[21]}$ ) 


\begin{tabular}{l|c|c|c} 
& "DND" & "DND_AirOx" & "DND_AirOx_Acid*” \\
\hline Sodium 1s & 0.0 & 0.1 & 1.6 \\
Oxygen 1s & 6.2 & 13.9 & 12.6 \\
Nitrogen 1s & 1.5 & 1.7 & 1.6 \\
C=O & 0.6 & 1.6 & 1.8 \\
C-O, C-N & 2.3 & 5.8 & 5.5 \\
$s p^{3}$ & 44.1 & 71.9 & 73.9 \\
$s p^{2}$ & 44.2 & 0.0 & 0.1 \\
Graphite & 1.1 & 4.6 & 2.9 \\
Silicon 2p & 0.1 & 0.4 & 0.0
\end{tabular}

Table 2. Relative atomic concentrations (in \%) obtained from XPS measurements. The C1s spectra are shown in Figure S2. 


\section{Supporting Information}

\section{A simple and soft chemical deaggregation method producing single-digit detonation nanodiamonds}

Daiki Terada ${ }^{\dagger}$, Frederick T.-K. So ${ }^{\dagger}$, Bodo Hattendorf, Eiji Ōsawa, Masahiro Shirakawa, Ryuji Igarashi* and Takuya F. Segawa*

${ }^{\dagger}$ Equal author contribution

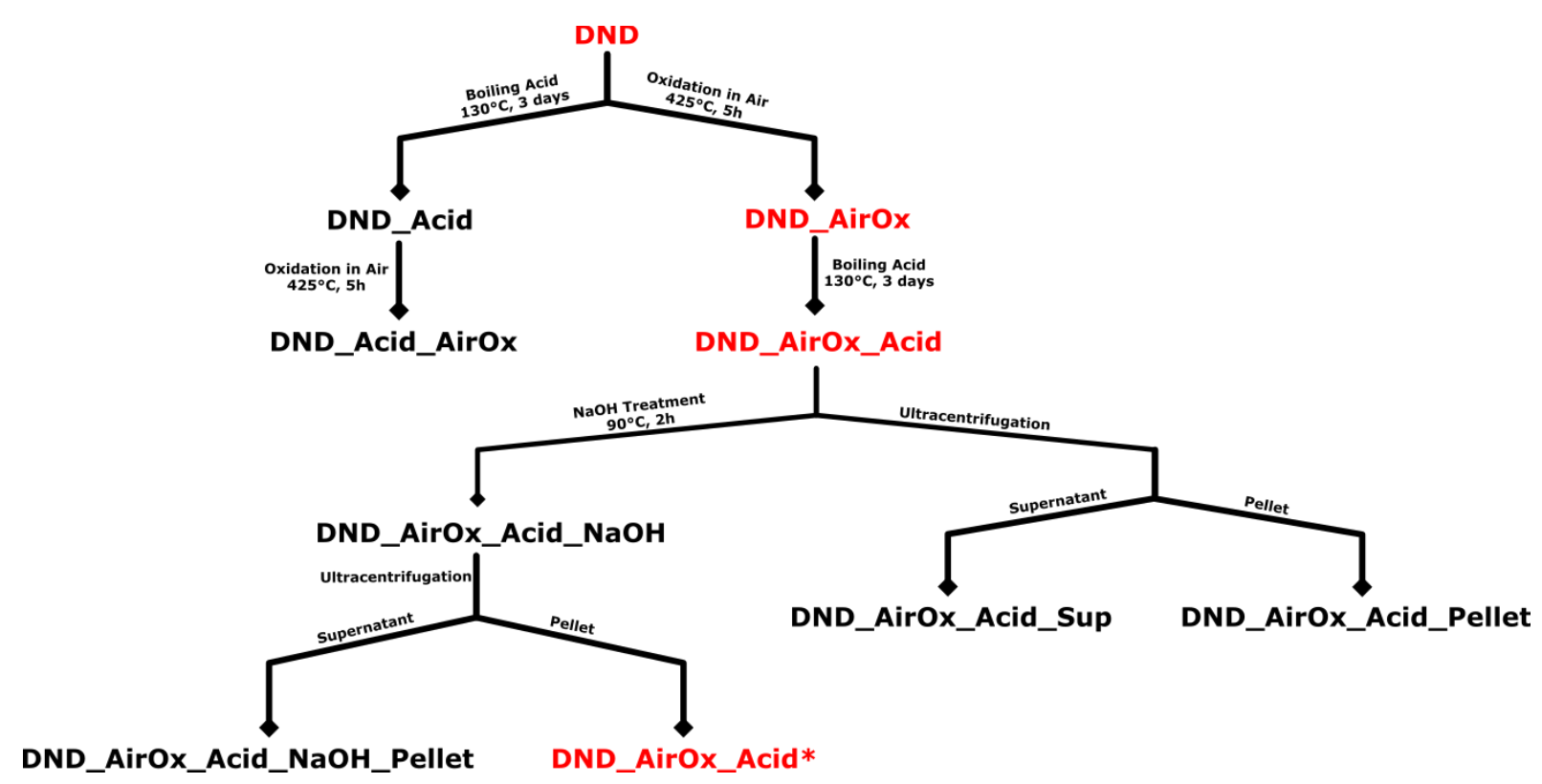

Scheme S1. Overview of all reaction steps and products. The red ones are the starting, intermediate and final products discussed in the main text. 


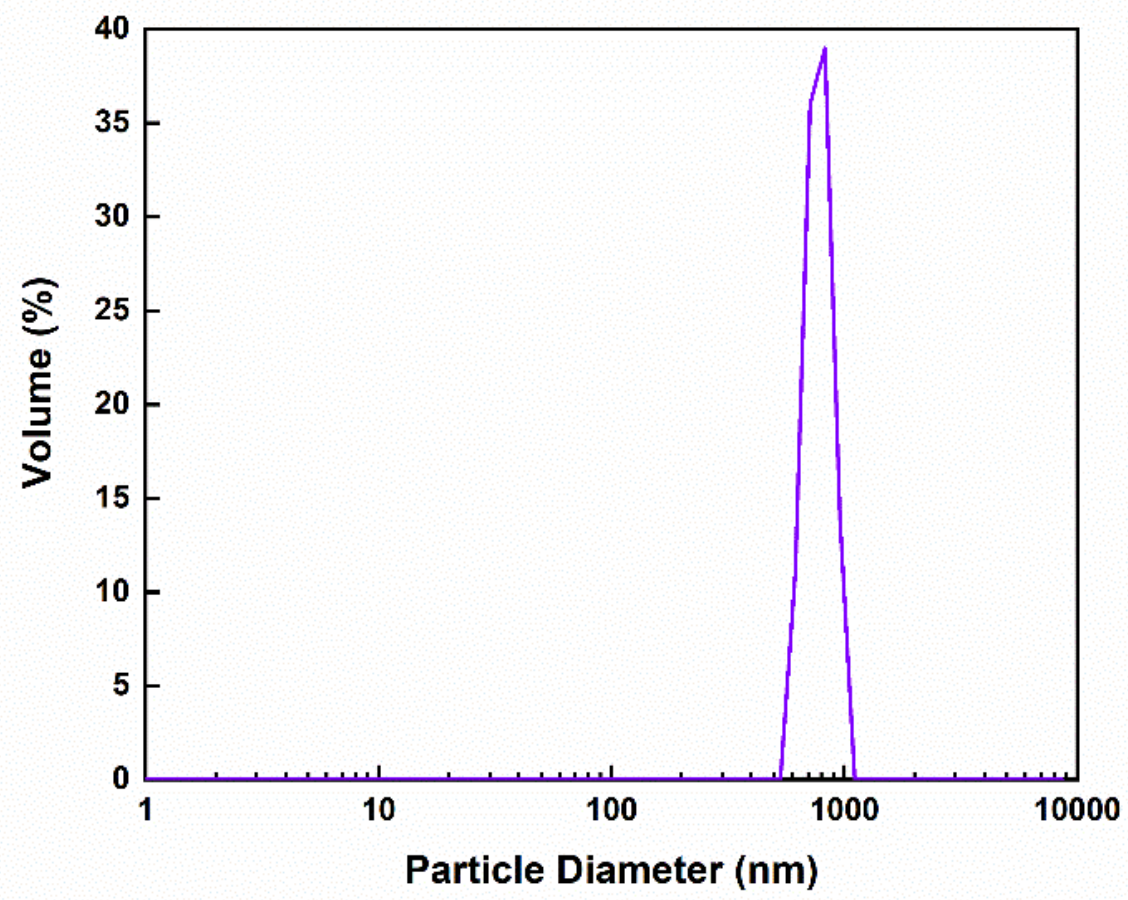

Figure S1. DLS size distribution for "DND_Acid" (see Scheme S1) by treatment of the starting "DND" with boiling acid only, where a dispersion cannot be reached. 

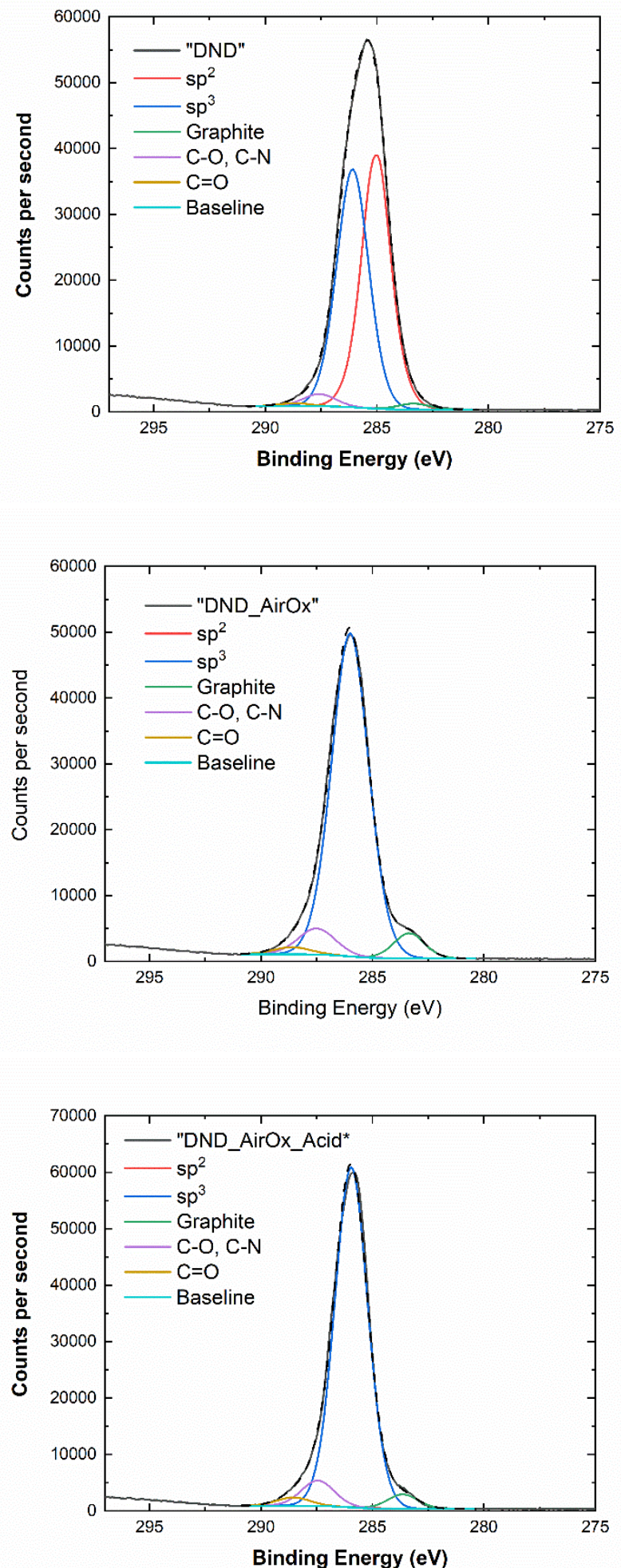

Figure S2. XPS C1s spectra of "DND", "DND_AirOx" and "DND_AirOx_Acid" with the individual fitting curves. 
2020-11-23

\section{LA-ICPMS}

This method allows for a rapid qualitative comparison of the respective ion signal intensities relative to ${ }^{13} \mathrm{C}$ as reference isotope. One needs to keep in mind however that these intensity ratios do not represent the mass fractions of the element in a sample because the isotopes' sensitivities in an ICPMS vary substantially because of a) isotope abundance, b) ionization efficiency and c) preferred transmission of heavier isotopes. The data can thus only be used to compare the relative abundance of a specific element across the different samples. The resulting intensity ratios are listed in Table S1. Each sample was analyzed at five randomly chosen positions with both MRP settings of the ICPMS. Listed are the mean intensity ratios from the repeat analyses and the resulting standard deviations together with the range observed.

The measurements revealed substantial heterogeneity within each sample not only for the different ablation spots but also within a particular depth profile. An example is given in Figure S3, showing the net ion signals for selected isotope during an ablation. One can observe distinct patterns in the ion signals. While the matrix isotope ${ }^{13} \mathrm{C}$ or trace elements as $\mathrm{Ba}$ and $\mathrm{Pb}$ do not show substantial variability above the noise throughout the ablation, $\mathrm{Ti}$ and $\mathrm{Na}$ (less obvious because of the higher base level) show an apparently correlated increase between 40 and 120 seconds of the analysis. This indicates that there is a substantial enrichment ( $\approx 10$ times for Ti in this case) of these elements over a depth of several $\mu \mathrm{m} . \mathrm{Zr}$ and $\mathrm{Hf}$ on the other hand exhibit a noisy signal structure characterized by numerous, short spikes. Such transient signals are typical for mineral grains of different size, which are present randomly distributed within the bulk. These elements accordingly show a greater spread also in the repeat analyses of the individual samples (Table S1). As a comparison the commercial NanoAmando (NanoCarbon Research Institute) DNDs were measured for comparison. Since 
their dispersion is perfomed by BASD using Zirconia beads, the $\mathrm{Zr}$ contamination is about two orders of magnitude higher than in our purely chemically dispersed samples.

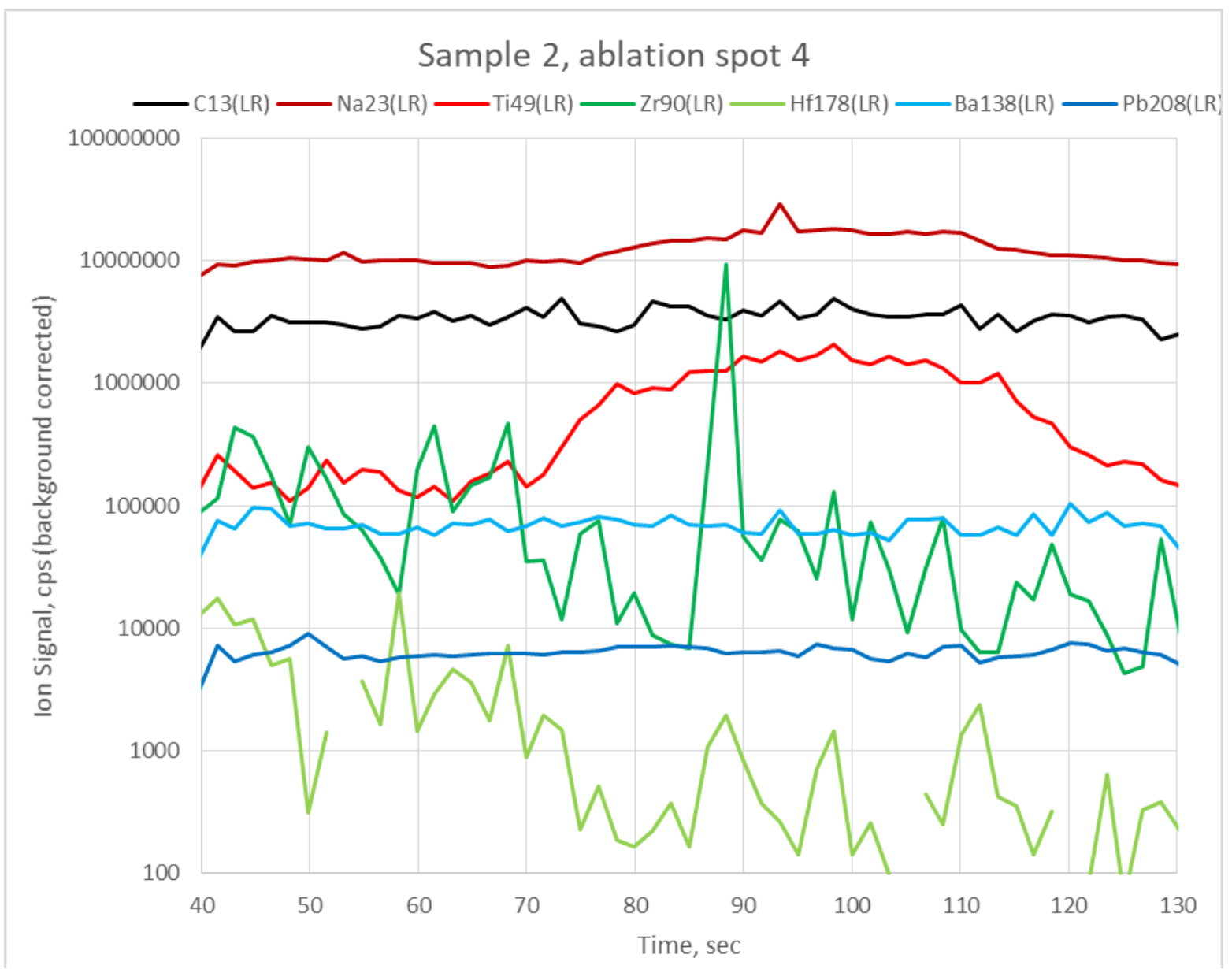

Figure S3. Example for a time resolved LA-ICPMS acquisition of a DND-sample. Smooth signals indicate a homogenous distribution of the elements (e.g. $\mathrm{Ba}, \mathrm{Pb}$ ) across the ablated depth, while gradual changes as visible for example in Ti are stem from large-scale heterogeneities. Short, random spikes in $\mathrm{Zr}$ and $\mathrm{Hf}$ on the other hand resemble individual (sub-) microns-sized individual particulates present in the material. The inhomogeneous signal could stem from the fact that the measured sample "DND_AirOx_Acid" (see Scheme S1) was not yet separated by ultracentrifugation. 
Table S1: Intensity ratios obtained by LA-ICPMS relative to ${ }^{13} \mathrm{C}$ as reference isotope

\#: net ion signal intensities $\left(10^{6} \mathrm{cps}\right),{ }^{*}$ : Medium resolution measurement (MRP=4000), n.d.: below limit of detection (LOD)

\begin{tabular}{|c|c|c|c|c|c|c|c|c|c|c|}
\hline & "DND" & & & & & "DND_Air & & & & \\
\hline & Mean & SD & $\max$ & $\min$ & LOD & Mean & SD & $\max$ & $\min$ & LOD \\
\hline${ }^{13} \mathrm{C} \#$ & 3.375 & 0.040 & 3.410 & 3.320 & & 3.16 & 0.13 & 3.28 & 3.01 & \\
\hline${ }^{13} \mathrm{C}^{*} \#$ & 0.304 & 0.016 & 0.321 & 0.287 & & 0.309 & 0.023 & 0.311 & 0.289 & \\
\hline${ }^{11} \mathrm{~B}$ & 0.009 & 0.003 & 0.014 & 0.0061 & 0.00015 & 0.021 & 0.001 & 0.023 & 0.021 & 0.00015 \\
\hline${ }^{23} \mathrm{Na}$ & 0.8 & 0.4 & 1.5 & 0.5 & 0.03 & 1.5 & 0.5 & 2.3 & 1.1 & 0.015 \\
\hline${ }_{*}^{24} \mathrm{Mg}$ & 0.159 & 0.018 & 0.174 & 0.132 & 0.0018 & 0.366 & 0.026 & 0.404 & 0.330 & 0.014 \\
\hline${ }^{27} \mathrm{Al}{ }^{*}$ & 0.38 & 0.08 & 0.47 & 0.29 & 0.044 & 0.91 & 0.14 & 1.06 & 0.74 & 0.044 \\
\hline${ }^{28} \mathrm{Si}^{*}$ & 1.45 & 0.16 & 1.60 & 1.22 & 0.008 & 3.27 & 0.21 & 3.53 & 2.95 & 0.009 \\
\hline${ }^{32} \mathrm{~S}^{*}$ & 0.274 & 0.026 & 0.309 & 0.240 & 0.009 & 0.664 & 0.023 & 0.680 & 0.622 & 0.009 \\
\hline${ }^{39} \mathrm{~K}$ & 0.13 & 0.05 & 0.17 & 0.05 & 0.018 & 0.36 & 0.04 & 0.40 & 0.31 & 0.008 \\
\hline${ }^{44} \mathrm{Ca}^{*}$ & 0.067 & 0.006 & 0.073 & 0.058 & 0.0015 & 0.143 & 0.007 & 0.144 & 0.136 & 0.0015 \\
\hline${ }^{49} \mathrm{Ti}$ & 0.09 & 0.09 & 0.21 & 0.026 & 0.00007 & 0.12 & 0.08 & 0.24 & 0.04 & 0.0001 \\
\hline${ }^{51} \mathrm{~V}^{*}$ & 0.0066 & 0.0020 & 0.0091 & 0.0054 & 0.00025 & 0.027 & 0.008 & 0.040 & 0.021 & 0.00027 \\
\hline${ }^{52} \mathrm{Cr}^{*}$ & 4.8 & 0.5 & 5.7 & 4.4 & 0.0015 & 9.5 & 0.4 & 9.7 & 8.8 & 0.0024 \\
\hline${ }^{55} \mathrm{Mn}$ & 0.22 & 0.05 & 0.30 & 0.16 & 0.00021 & 0.51 & 0.05 & 0.52 & 0.45 & 0.00028 \\
\hline${ }^{57} \mathrm{Fe}$ & 0.047 & 0.007 & 0.056 & 0.038 & 0.0007 & 0.104 & 0.007 & 0.116 & 0.093 & 0.0006 \\
\hline${ }^{57} \mathrm{Co}$ & n.d. & & & & 0.0008 & 0.00107 & 0.00028 & 0.00130 & 0.00068 & 0.0008 \\
\hline${ }^{60} \mathrm{Ni}$ & 0.0008 & 0.0003 & 0.0014 & 0.0006 & 0.00015 & 0.0015 & 0.0004 & 0.0019 & 0.0012 & 0.0002 \\
\hline${ }^{63} \mathrm{Cu}$ & 0.028 & 0.006 & 0.034 & 0.019 & 0.0002 & 0.066 & 0.006 & 0.0723 & 0.060 & 0.00025 \\
\hline${ }^{68} \mathrm{Zn}$ & 0.0043 & 0.0006 & 0.0053 & 0.0040 & 0.0004 & 0.0102 & 0.0006 & 0.0112 & 0.0095 & 0.0006 \\
\hline${ }^{90} \mathrm{Zr}$ & 0.013 & 0.005 & 0.020 & 0.009 & 0.00002 & 0.072 & 0.026 & 0.093 & 0.0063 & 0.00004 \\
\hline${ }^{118} \mathrm{Sn}$ & 0.0037 & 0.0005 & 0.0042 & 0.0030 & 0.00007 & 0.0073 & 0.0006 & 0.0079 & 0.0071 & 0.00007 \\
\hline${ }^{121} \mathrm{Sb}$ & 0.00137 & 0.00017 & 0.00155 & 0.00109 & 0.00001 & 0.00287 & 0.00018 & 0.00309 & 0.00264 & 0.00001 \\
\hline${ }^{138} \mathrm{Ba}$ & 0.0112 & 0.0017 & 0.0141 & 0.0100 & 0.00005 & 0.0230 & 0.0011 & 0.0237 & 0.0216 & 0.00006 \\
\hline${ }^{178} \mathrm{Hf}$ & 0.00021 & 0.00006 & 0.00031 & 0.00015 & 0.000006 & 0.0008 & 0.0003 & 0.0012 & 0.0005 & 0.00001 \\
\hline${ }^{184} \mathrm{~W}$ & 0.007 & 0.005 & 0.016 & 0.003 & 0.000004 & 0.0107 & 0.0014 & 0.0121 & 0.0084 & 0.000003 \\
\hline${ }^{208} \mathrm{~Pb}$ & 0.00102 & 0.00016 & 0.00118 & 0.00075 & 0.000007 & 0.00205 & 0.00015 & 0.00222 & 0.00184 & 0.000008 \\
\hline
\end{tabular}




\begin{tabular}{|c|c|c|c|c|c|c|c|c|c|c|}
\hline & \multicolumn{5}{|c|}{ "DND_AirOx_Acid" } & \multicolumn{5}{|c|}{ NanoAmando (NanoCarbon Research Institute) } \\
\hline & Mean & SD & $\max$ & $\min$ & LOD & Mean & SD & $\max$ & $\min$ & LOD \\
\hline${ }^{13} \mathrm{C} \#$ & 4.14 & 0.29 & 4.61 & 4.03 & & 3.85 & 0.09 & 3.99 & 3.79 & \\
\hline${ }^{13} C^{*} \#$ & 0.370 & 0.017 & 0.392 & 0.344 & & 0.313 & 0.023 & 0.347 & 0.295 & \\
\hline${ }^{11} \mathrm{~B}$ & 0.0352 & 0.0027 & 0.0390 & 0.0337 & 0.0001 & 0.0217 & 0.0007 & 0.0222 & 0.0218 & 0.00015 \\
\hline${ }^{23} \mathrm{Na}$ & 0.046 & 0.026 & 0.084 & 0.029 & 0.01 & n.d. & & & & 0.01 \\
\hline${ }^{24} \mathrm{Mg}^{*}$ & n.d. & & & & 0.01 & n.d. & & & & 0.01 \\
\hline${ }^{27} \mathrm{Al}^{*}$ & 0.038 & 0.012 & 0.052 & 0.030 & 0.03 & 1.48 & 0.13 & 1.70 & 1.40 & 0.03 \\
\hline${ }^{28} \mathrm{Si}^{*}$ & 3.2 & 0.3 & 3.7 & 2.9 & 0.007 & 0.19 & 0.06 & 0.25 & 0.19 & 0.008 \\
\hline${ }^{32} \mathrm{~S}^{*}$ & 0.010 & 0.005 & 0.101 & 0.094 & 0.007 & 0.076 & 0.016 & 0.10 & 0.066 & 0.007 \\
\hline${ }^{39} \mathrm{~K}$ & 0.014 & 0.006 & 0.019 & 0.012 & 0.006 & n.d. & & & & 0.006 \\
\hline${ }^{44} \mathrm{Ca}^{*}$ & 0.0021 & 0.0008 & 0.0033 & 0.0018 & 0.0008 & 0.0020 & 0.0012 & 0.0040 & 0.0009 & 0.0009 \\
\hline${ }^{49} \mathrm{Ti}$ & 0.109 & 0.008 & 0.121 & 0.105 & 0.0001 & 0.0055 & 0.0011 & 0.0067 & 0.0048 & 0.0001 \\
\hline${ }^{51} \mathrm{~V}^{*}$ & 0.0200 & 0.0015 & 0.0218 & 0.0186 & 0.00021 & 0.0019 & 0.0005 & 0.0027 & 0.0015 & 0.0002 \\
\hline${ }^{52} \mathrm{Cr}^{*}$ & 0.928 & 0.029 & 0.960 & 0.899 & 0.0024 & 0.09 & 0.06 & 0.14 & 0.032 & 0.0018 \\
\hline${ }^{55} \mathrm{Mn}$ & 0.0085 & 0.0021 & 0.0118 & 0.0083 & 0.00017 & 0.0077 & 0.0009 & 0.0089 & 0.0069 & 0.0002 \\
\hline${ }^{57} \mathrm{Fe}$ & 0.0075 & 0.0023 & 0.0113 & 0.0058 & 0.0004 & 0.0227 & 0.0012 & 0.0238 & 0.0224 & 0.0004 \\
\hline${ }^{57} \mathrm{Co}$ & n.d. & & & & 0.0005 & n.d. & & & & 0.0005 \\
\hline${ }^{60} \mathrm{Ni}$ & 0.0005 & 0.0003 & 0.0008 & 0.0003 & 0.00015 & 0.0023 & 0.0005 & 0.0031 & 0.0017 & 0.00015 \\
\hline${ }^{63} \mathrm{Cu}$ & 0.0016 & 0.0007 & 0.0026 & 0.0010 & 0.0002 & 0.00136 & 0.00015 & 0.00161 & 0.00133 & 0.0002 \\
\hline${ }^{68} \mathrm{Zn}$ & n.d. & & & & 0.0005 & n.d. & & & & 0.0005 \\
\hline${ }^{90} \mathrm{Zr}$ & 0.030 & 0.024 & 0.055 & 0.012 & 0.000025 & 6.92 & 0.29 & 7.27 & 6.76 & 0.00005 \\
\hline${ }^{118} \mathrm{Sn}$ & 0.00254 & 0.00019 & 0.00281 & 0.00247 & 0.00006 & 0.00143 & 0.00008 & 0.00152 & 0.00134 & 0.00006 \\
\hline${ }^{121} \mathrm{Sb}$ & 0.00251 & 0.00021 & 0.00285 & 0.00234 & 0.00001 & 0.000215 & 0.000014 & 0.000230 & 0.000208 & 0.00001 \\
\hline${ }^{138} \mathrm{Ba}$ & 0.0283 & 0.0028 & 0.0325 & 0.0257 & 0.00004 & 0.0019 & 0.0003 & 0.0024 & 0.0018 & 0.00005 \\
\hline${ }^{178} \mathrm{Hf}$ & 0.00033 & 0.00020 & 0.00056 & 0.00018 & 0.00001 & 0.0820 & 0.0012 & 0.0838 & 0.0809 & 0.000003 \\
\hline${ }^{184} \mathrm{~W}$ & 0.0051 & 0.0003 & 0.0055 & 0.0049 & 0.000002 & 0.0940 & 0.0007 & 0.0947 & 0.0928 & 0.000005 \\
\hline${ }^{208} \mathrm{~Pb}$ & 0.00014 & 0.00004 & 0.00020 & 0.00012 & 0.000007 & 0.000520 & 0.000019 & 0.000540 & 0.000509 & 0.000006 \\
\hline
\end{tabular}

